\title{
Determinants of School Education of Scheduled Tribes in Eastern Uttar Pradesh with Special Reference to their Socio-economic Status
}

\section{Poonam Singh Kharwar ${ }^{1 *}$, Abhishek Kumar ${ }^{2}$ and Abhinav Kumar ${ }^{3}$}

\author{
${ }^{1}$ Assistant Professor, Banaras Hindu University, Uttar Pradesh, India \\ ${ }^{2}$ Research Scholar, Indian Institute of Technology, Kharagpur, West Bengal, India \\ ${ }^{3}$ DM Cardiology, AIIMS, Bhubneshwar, Odisha, India
}

*Corresponding author: poonam.kharwar3@gmail.com

Received: 10 Sept., 2021

Revised: 24 Nov., 2021

Accepted: 11 Dec., 2021

\begin{abstract}
Educational development plays a vital role for the socio-economic betterment of the weaker sections of the society. Tribes residing in eastern Uttar Pradesh are still very poor in education in spite of the Government's commitments to improve their backward status through special constitutional provision. Present article analyzes the determinants of school education of Scheduled Tribes with special reference to their socio-economic status as major determinant and explores possible strategies for improvement of different ST communities in Deoria, Ballia, Ghazipur, Varanasi and Sonbhadra district of U.P. Most of the husband or wife (55.9\%) in ST families are primary to $10+2$ educated or illiterate $(31.4 \%)$ and, $12.2 \%$ of families are still not sending their children to school. Poor socio-economic status due to several contributing factors is the biggest hurdle in getting higher education in tribes. Most of them are either poor $(73.4 \%)$ or lower middle (24\%) socio-economic grade even $96.8 \%$ poor in Sonbhadra, most of them earn their livelihood from irregular mostly agriculture labour (83.82\%), only $2.4 \%$ has farming resulting poor yield of their small inadequate land with traditional manner of farming. Poor percentage of children studying in residential school, school away from easy approach, lack of convenience, less availability of time, poor school facilities and student friendly environment, poor parental attitude and support are other determinants resulting poor educational outcome in children. Poor personal hygiene among children also predisposes them to discrimination in school environment. Corrective measures against identified determinants needs further strengthening to improve overall status of tribes.
\end{abstract}

Keywords: Tribes, socio-economic status, determinants of education, personal hygiene

How to cite this article: Kharwar, P.S., Kumar, A. and Kumar, A. (2021). Determinants of School Education of Scheduled Tribes in Eastern Uttar Pradesh with Special Reference to their Socio-economic Status. Learning Community, 12(02): 73-93. 
India has the largest concentration of tribal people anywhere in the world (except in Africa). The areas inhabited by the tribal constitute significant part of the under-developed areas of the country as society segregate these tribes from the general people in various respects. Article 366 (25) of the Constitution of India defined 'scheduled tribes' as "such tribes or tribal communities or parts of or groups within such tribes or tribal communities as are deemed under Article 342 to be Scheduled Tribes (ST) for the purposes of this constitution". There is need for equitable and balanced progress of all sections of human communities for the development of a society and for this perspective, so it is imperative to bring the weaker, deprived and discriminated sections such as STs in India to the mainstream of national development. The Government of India is under constitutional obligation to protect the interests of these communities and to uplift them socially and economically. Uttar Pradesh (U.P.) is home to a large population cut off from the mainstream of development. It is one of the least developed states with a Human Development Index value of 0.380 (2007-08) which is lower than the national average of 0.467 making it stand at $18^{\text {th }}$ rank among Indian states/UTs. Eastern U.P. accounts for 39.98\% of total U.P. population, which has highest population density in the state. U.P. has traditionally been center of diverse tribes and, during 1967, five tribes namely Tharu, Buksa, Janusari, Bhotia and Raji were declared ST under Article 342 by the then President of India which constituted $0.6 \%$ of total population of U.P. At present, there are 15 notified tribal communities in U.P. but welfare programmes directed for the development of these STs have not resulted any visible positive impact. Given the common backwardness and suffering of ST population in eastern U.P., it is essential to understand and identify the underlying correlates, which place their life to very miserable conditions. Educational development plays a vital role for the socio-economic betterment of the weaker sections of the society. Population of STs is less in eastern U.P. so it has not drawn attention of researchers in past. No extensive field study has been reported of STs in socio-economically backward region eastern U.P. hence there is an urgent need to conduct such study to fill up the gap of knowledge and to provide guidelines and strategies for formulation of sustainable development programme for the overall betterment of these deprived community. Present study was conducted among families of different ST communities in Deoria, Ballia, Ghazipur, Varanasi and Sonbhadra district of U.P. with following objectives:

1. To study the status of education of STs in eastern U.P.

2. To study the socio-economic status and livelihood profile of STs in eastern U.P.

3. To study the status of other determinants of school education of STs in eastern U.P.

4. To study personal hygiene profile of STs in eastern U.P.

5. To explore the further possibilities to strengthen educational status of STs in eastern U.P.

\section{Review of Literature}

Tribes have existed at the margins of Hindu civilization. Hindu civilization acknowledged the distinction between tribe and caste in the distinction between two kinds of communities Jan an Jati where one confined to the isolation of hills and forests and, other settled in villages and towns with a more elaborate 
division of labours. U.P. has traditionally been centre of diverse tribes. During 1967, five tribes namely Tharu, Buksa, Janusari, Bhotia and Raji were declared ST under Article 342 by the then President of India which constituted $0.6 \%$ of total population of U.P. As per the Census 2011, the tribes constitute around $8.6 \%$ of the total Indian population and $89.97 \%$ of them live in rural areas. U.P. has the lowest $(0.57 \%)$ proportion of ST population. U.P. stands $17^{\text {th }}$ in terms of number of STs among all the states in India. Approximately $84 \%$ of the total population lived in eastern part of U.P. The tribal population of Sonbhadra is highest (20.7\%) in the state higher than the national average of $8.6 \%$; tribal population was less than $0.1 \%$ of total population in 33 out of total 71 districts located in other than the eastern region. At present, there are 15 notified tribal communities in U.P. Gond along with the sub-ethnic groups accounts for $50.2 \%$ of tribes of all STs, Kharwar contribute $14.6 \%$ of total ST population of state. Tharu is the third largest community, its population growth from of $26 \%$ from 83,544 from in 2001 to 1,05,291 in 2011 and their percentage share in all STs has decreased from 77.4 in 2001 to 9.3 in 2011 due to reorganization of U.P. state. Saharya accounts $6.25 \%$ of all STs followed by Chero (3.7\%). Thus according to census 2011, all these five tribes constitute $83.6 \%$ of ST population of U.P. Baiga and Pankha/Panika constituted 1.5 and $1.4 \%$ respectively. Agariya and Bhuiya/Bhuinya constituted 2.6 and $2.2 \%$ respectively. Population share of Bhotia (0.5\%), Buksa $(0.4 \%)$, Janusari $(0.3 \%)$, Raji $(0.1 \%)$, Parahiya $(0.1 \%)$ and, Patari $(0.01 \%)$ contributes minimum in ST population. (Government of India, 2013).

Belshaw (1972) observed that though a lot has been done for tribal's social and economic betterment, yet a great deal remains to be done. Gare (1983) presented that extremely low levels of literacy, education, health and nutrition status, inadequate unemployment and unproductive agriculture of tribal put a constraint on improving their socio-economic status. Aphale and Bairagi (1984) also pointed that high percentage of disparity among the tribal are due to their backwardness, ignorance and poverty.

Singh (1997) mentioned that the colonial period mentioned the transformation of forest dwellers into the tribes and later on into the castes but distinction between tribes and castes fully emerged during census of 1901. It was under the government of India Act of 1935 and the Constitution of India that the nomenclature of the ST fully emerged. Tribal in India is the most adversely affected ethnic group due to the development in post-independence India, development should not be studied in isolation, as it is not synonymous of with the growth of few affluent persons. Amartya Sen (1999) stated unless the capabilities among human beings are adequately addressed and deprivations faced by marginalized groups are overcome, development can not take place. Rai (2017) found economic situation of ST at lowest level in the society. Rai (2018) mentioned that tribes of U.P. are living in conditions of deprivation; their economic condition and standard of living are very low, as most of them don't have land, asset and education.

Illiteracy is a major educational problem of the tribes as also reported by NSSO (1991), literacy reported to be only $4.1 \%$ by Basu (2000) among females in Rajasthan and $55.7 \%$ among tribes of U.P. less than national average by Government of India (2013). School dropout rate has been very high in ST. Jha and Jhingan (2002) mentioned that regular school attendance is a problem in the tribal areas, further aggravated by teacher absenteeism. Jayaswal et. al. (2003) found that parents of lower achievers 
were not strongly ambitious of children's upward mobility. Hasnain (2004) reported education to be a matter of luxury for the deprived tribal family. Burman (2009) pointed out that only $88.46 \%$ of ST households are covered by primary schools in a radius of $1 \mathrm{~km}$. Pradhan (2011) described that despite special initiatives like Ashram schools, introducing vernacular at primary level, and teaching in local dialects, the tribal are still lagging behind the non-tribal. Ambuselvi et al. (2018) reported that majority of the tribal children were engaged in crucial family work which deprive them to spare adequate time for study and boost their school absenteeism tendency. Residential schools are very poor in infrastructural facilities and have poor hygiene and poor noon meal program.

Human Rights Watch (2014) records prejudice of adverse situations perceived by teachers along with poor personal hygiene of tribal children as factors responsible for obstacles in teaching and learning process. Jaiswal (2017) mentioned that old people and children generally avoid bathing and use alav in winter season leading to foul smell.

\section{Methodology}

All ST communities living in eastern U.P. comprised as universe of the study. There are 15 notified tribal communities in U.P. Five districts of eastern U.P. namely Sonbhadra, Varanasi, Ghazipur, Deoria, and Ballia were selected randomly to conduct the study. A semi-constructed questionnaire prepared by the author was used which was based on viewpoints regarding indicators of general health, education and socio-economic status with the help of health expert and other related experts. Section A has 22 questions related to socio economic status constructed by O.P. Aggarwal, S.K. Bhasin, A.K. Sharma, P. Chhabra, K. Aggarwal, and O.P. Rajoura (2005), a scale suitable for all sections of society. Section $\mathrm{B}$ has questions related to general health and education status. Section $\mathrm{C}$ has questions related to other determinants associated with general health, education \& socio-economic conditions. Present research project being extensive field study was performed by survey research method based on the primary as well as secondary data collected by observation and interview. Field surveyors used the semi-constructed questionnaire to collect the data from the study sample, which consists of selected 11416 families residing in 474 villages of five districts. Field surveyors also subjected them to scheduled information interview and observation techniques as per need. The secondary data were collected from the relevant published documents. Data were collected, compiled in Excel sheet of SPSS version 16, analyzed and subjected to vigorous statistical treatment for analysis as per need.

\section{RESULTS}

\section{Status of education of STs in eastern U.P.}

\section{Education of husband or wife}

Table 1 presents education status of better-educated husband or wife in the ST family. It reveals that maximum numbers $(55.9 \%)$ of husband or wife in ST families in all of the selected five districts are 
Determinants of School Education of Scheduled Tribes in Eastern Uttar Pradesh...

primary to $10+2$ educated followed by illiteracy (31.4\%). Only $4.9 \%$ of husband or wife in families is graduate or more educated.

Table 1: Education of Husband or wife in ST families

\begin{tabular}{|c|c|c|c|c|c|c|}
\hline \multirow{2}{*}{ Education level } & \multirow{2}{*}{$\begin{array}{l}\text { Total no. of } \\
\text { families }(\%)\end{array}$} & \multicolumn{5}{|c|}{ District wise distribution: number (\%) } \\
\hline & & Sonbhadra & Varanasi & Ghazipur & Deoria & Ballia \\
\hline Illiterate & $3583(31.4 \%)$ & $389(20.7 \%)$ & $\begin{array}{l}1013 \\
(42.5 \%)\end{array}$ & $864(35.9 \%)$ & $\begin{array}{l}1102 \\
(41.6 \%)\end{array}$ & $215(10.3 \%)$ \\
\hline $\begin{array}{l}\text { Just literate but no } \\
\text { schooling }\end{array}$ & $275(2.4 \%)$ & $115(6.1 \%)$ & $43(1.8 \%)$ & $57(2.4 \%)$ & $49(1.8 \%)$ & $11(0.5 \%)$ \\
\hline Primary attended & $654(5.7 \%)$ & $269(14.3 \%)$ & $83(3.5 \%)$ & $129(5.4 \%)$ & $171(6.5 \%)$ & $2(0.1 \%)$ \\
\hline $\begin{array}{l}\text { Primary pass to } \\
\text { less than 10th }\end{array}$ & $2358(20.7 \%)$ & $369(19.6 \%)$ & $526(22 \%)$ & $491(20.4 \%)$ & $686(25.9 \%)$ & $286(13.7 \%)$ \\
\hline $\begin{array}{l}10^{\text {th }} \text { pass to less } \\
\text { than graduate }\end{array}$ & $4033(35.3 \%)$ & $730(38.8 \%)$ & $537(22.5 \%)$ & $713(29.6 \%)$ & $543(20.5 \%)$ & $1510(72.2 \%)$ \\
\hline Graduation & $414(3.6 \%)$ & $5(0.3 \%)$ & $151(6.3 \%)$ & $125(5.2 \%)$ & $85(3.9 \%)$ & $48(2.3 \%)$ \\
\hline $\begin{array}{l}\text { Post graduate } \\
\text { nontechnical } \\
\text { including Ph.D. }\end{array}$ & $79(0.7 \%)$ & $3(0.1 \%)$ & $27(1.1 \%)$ & $29(1.2 \%)$ & $14(0.5 \%)$ & $6(0.3 \%)$ \\
\hline $\begin{array}{l}\text { Professional } \\
\text { qualified }\end{array}$ & $20(0.2 \%)$ & 0 & $6(0.3 \%)$ & 0 & 0 & $14(0.7 \%)$ \\
\hline Total & 11416 & 1880 & 2386 & 2408 & 2650 & 2092 \\
\hline
\end{tabular}

\section{Education of children}

Table 2 presents education of children in ST families in study districts. It shows that maximum number $(42.3 \%)$ of ST families are sending or have sent their all children to school, followed by $39.2 \%$ families sending or have sent their $>50 \%$ children to school. On average $12.2 \%$ of families are still not sending any children to school. Sonbhadra ST families have dismal records of child education, $21.1 \%$ of them are not sending any children to school and 3.2\% of them have never sent their all children. Majority of ST children goes to schools mainly during admission season to get the free bag and only at the time of mid-day meal distribution, a surprising fact noted at all places.

Table 2: Education of children in ST families

\begin{tabular}{lllllll}
\hline Education of children in & Total no. of & \multicolumn{4}{c}{ District wise distribution: number (\%) } \\
\cline { 3 - 6 } family & families (\%) & Sonbhadra & Varanasi & Ghazipur & Deoria & Ballia \\
\hline All children going/ever & $4831(42.3 \%)$ & $72(3.8 \%)$ & $480(20.1 \%)$ & 1525 & 1337 & 1417 \\
gone to school & & & & $(63.3 \%)$ & $(50.5 \%)$ & $(67.7 \%)$ \\
$>50 \%$ children ever gone// & $4474(39.2 \%)$ & $1333(70.9 \%)$ & 1496 & $430(17.9 \%)$ & 892 & 323 \\
going to school & & & $(62.7 \%)$ & & $(33.7 \%)$ & $(13.5 \%)$
\end{tabular}




\begin{tabular}{lllllll}
$<50 \%$ children ever & $716(6.3 \%)$ & $79(4.2 \%)$ & $251(10.5 \%)$ & $173(7.2 \%)$ & 144 & $69(3.3 \%)$ \\
$\begin{array}{l}\text { gone/going to school } \\
\begin{array}{l}\text { No child ever gone/going } \\
\text { to school }\end{array}\end{array}$ & $1395(12.2 \%)$ & $396(21.1 \%)$ & $159(6.7 \%)$ & $280(11.6 \%)$ & $\begin{array}{l}277 \\
(10.4 \%)\end{array}$ & 283 \\
\hline Total & & & & & $\mathbf{2 3 0 . 5 \%}$ & $(13.5 \%)$ \\
\hline
\end{tabular}

\section{Socio-economic status and livelihood profile of STs in eastern U.P.}

\section{Socio-economic grades}

Analysis of different socio-economic grades (upper high, high, upper middle, lower middle, poor and very poor) of ST families in study districts is presented in Table 3. It reveals that maximum numbers (73.4\%) of ST family in all of the selected five districts are in poor grade, which is highest $(96.8 \%)$ in Sonbhadra and lowest $(51.5 \%)$ in Ghazipur. It is followed by lower middle-income grade $(24 \%)$ on average, which is highest (45.7\%) in Ghazipur and lowest $(0.3 \%)$ in Sonbhadra. None of them are in upper grade and almost negligible $(0.09 \%)$ in high grade, only $1.5 \%$ of them are in upper middle grade, $0.3 \%$ to $3 \%$ of are very poor. ST families living in Ghazipur are better in socio-economic grades than those living in Sonbhadra.

Table 3: Socio-economic grade of families

\begin{tabular}{|c|c|c|c|c|c|c|c|}
\hline \multirow{2}{*}{ Grade } & \multirow{2}{*}{ Scale } & \multirow{2}{*}{$\begin{array}{l}\text { Total no. of } \\
\text { families }(\%)\end{array}$} & \multicolumn{5}{|c|}{ District wise distribution: number (\%) } \\
\hline & & & Sonbhadra & Varanasi & Ghazipur & Deoria & Ballia \\
\hline Very poor & $0-15$ & $120(1.1 \%)$ & $56(3 \%)$ & $27(1.1 \%)$ & $7(0.3 \%)$ & $\begin{array}{l}12 \\
(0.5 \%)\end{array}$ & $\begin{array}{l}18 \\
(0.9 \%)\end{array}$ \\
\hline Poor & $16-30$ & $8383(73.4 \%)$ & $\begin{array}{l}1819 \\
(96.8 \%)\end{array}$ & $\begin{array}{l}1919 \\
(80.4 \%)\end{array}$ & $\begin{array}{l}1240 \\
(51.5 \%)\end{array}$ & $\begin{array}{l}1667 \\
(62.9 \%)\end{array}$ & $\begin{array}{l}1738 \\
(83.1 \%)\end{array}$ \\
\hline $\begin{array}{l}\text { Lower } \\
\text { middle }\end{array}$ & $31-45$ & $2739(24 \%)$ & $5(0.3 \%)$ & $\begin{array}{l}409 \\
(17.2 \%)\end{array}$ & $\begin{array}{l}1100 \\
(45.7 \%)\end{array}$ & $\begin{array}{l}910 \\
(34.3 \%)\end{array}$ & $\begin{array}{l}315 \\
(15.1 \%)\end{array}$ \\
\hline $\begin{array}{l}\text { Upper } \\
\text { middle }\end{array}$ & $46-60$ & $170(1.5 \%)$ & 0 & $31(1.3 \%)$ & $59(2.5 \%)$ & $\begin{array}{l}59 \\
(2.2 \%)\end{array}$ & $21(1 \%)$ \\
\hline High & $61-75$ & $4(0.09 \%)$ & 0 & 0 & $2(0.1 \%)$ & $2(0.1 \%)$ & 0 \\
\hline Upper high & $76 \&$ more & 0 & 0 & 0 & 0 & 0 & 0 \\
\hline Total & & 11416 & 1880 & 2386 & 2408 & 2650 & 2092 \\
\hline
\end{tabular}

\section{Livelihood profile}

Table 4 presents the livelihood profile of families. These families are primarily agrarian and most of them earn their livelihood from irregular labour (83.82\%), only $1.67 \%$ has sufficient and $0.73 \%$ insufficient farming. Small shop, occupation, service contributes $2 \%$ earnings equally from insufficient and sufficient amounts which is lowest (0.32\%) in Sonbhadra. Majority of ST families are poor but $47.7 \%$ of them 
Determinants of School Education of Scheduled Tribes in Eastern Uttar Pradesh...

don't have BPL card, 36.79\% possess BPL card but not benefitted by welfare schemes, $8.27 \%$ are card holders and get benefits and $1.81 \%$ possess card but suffer due to diseases and addiction. Prosperous people manage to get BPL card $(5.36 \%)$ which is lowest $(0.43 \%)$ in Sonbhadra.

Table 4: Livelihood profile of ST families

\begin{tabular}{|c|c|c|c|c|c|c|}
\hline \multirow{2}{*}{ Parameters of livelihood } & \multirow{2}{*}{$\begin{array}{l}\text { Number of } \\
\text { family }(\%)\end{array}$} & \multicolumn{5}{|c|}{ District wise distribution: number (\%) } \\
\hline & & Sonbhadra & Varanasi & Ghazipur & Deoria & Ballia \\
\hline \multicolumn{7}{|l|}{ Source of livelihood } \\
\hline None & $1091(9.55 \%)$ & $42(2.23 \%)$ & $\begin{array}{l}313 \\
(13.12 \%)\end{array}$ & $375(15.57 \%)$ & $\begin{array}{l}330 \\
(12.45 \%)\end{array}$ & $31(1.48 \%)$ \\
\hline Irregular labour & $\begin{array}{l}9569 \\
(83.82 \%)\end{array}$ & $\begin{array}{l}1734 \\
(92.23 \%)\end{array}$ & $\begin{array}{l}2025 \\
(84.87 \%)\end{array}$ & $\begin{array}{l}1788 \\
(74.25 \%)\end{array}$ & $\begin{array}{l}2230 \\
(84.15 \%)\end{array}$ & $\begin{array}{l}1792 \\
(85.56 \%)\end{array}$ \\
\hline Regular labour & 0 & 0 & 0 & 0 & 0 & 0 \\
\hline Insufficient farming & $84(0.73 \%)$ & $56(2.98 \%)$ & $9(0.38 \%)$ & $7(0.29 \%)$ & $12(4.53 \%)$ & 0 \\
\hline Sufficient farming & $191(1.67 \%)$ & $36(1.91 \%)$ & $7(0.29 \%)$ & $97(4.03 \%)$ & $13(4.9 \%)$ & $38(1.82 \%)$ \\
\hline $\begin{array}{l}\text { Insufficient small shop, } \\
\text { occupation, service }\end{array}$ & $245(2.15 \%)$ & $6(0.32 \%)$ & $22(0.92 \%)$ & $97(4.03 \%)$ & $30(1.13 \%)$ & $90(4.3 \%)$ \\
\hline $\begin{array}{l}\text { Sufficient small shop, } \\
\text { occupation, service }\end{array}$ & $232(2.03 \%)$ & $6(0.32 \%)$ & $10(0.42 \%)$ & $42(1.74 \%)$ & $33(1.24 \%)$ & $\begin{array}{l}141 \\
(6.74 \%)\end{array}$ \\
\hline $\begin{array}{l}\text { Big shop, occupation, } \\
\text { service }\end{array}$ & $4(0.03 \%)$ & 0 & 0 & $2(0.08 \%)$ & $2(0.07 \%)$ & 0 \\
\hline \multicolumn{7}{|l|}{ Livelihood status } \\
\hline $\begin{array}{l}\text { Poor but without BPL } \\
\text { card }\end{array}$ & $5446(47.7 \%)$ & $\begin{array}{l}1484 \\
(78.93 \%)\end{array}$ & $\begin{array}{l}1483 \\
(62.15 \%)\end{array}$ & $763(31.98 \%)$ & $\begin{array}{l}507 \\
(19.13 \%)\end{array}$ & $\begin{array}{l}1209 \\
(57.76 \%)\end{array}$ \\
\hline $\begin{array}{l}\text { Poor \&BPL card holder } \\
\text { but not benefitted by } \\
\text { welfare schemes }\end{array}$ & $\begin{array}{l}4199 \\
(36.79 \%)\end{array}$ & $16(0.85 \%)$ & $790(33.1 \%)$ & $\begin{array}{l}1333 \\
(55.87 \%)\end{array}$ & $\begin{array}{l}1953 \\
(73.7 \%)\end{array}$ & $\begin{array}{l}107 \\
(5.11 \%)\end{array}$ \\
\hline $\begin{array}{l}\text { BPL card holder but poor } \\
\text { due to addiction }\end{array}$ & $207(1.81 \%)$ & $2(0.11 \%)$ & $18(0.75 \%)$ & $26(1.09 \%)$ & $41(1.55 \%)$ & $\begin{array}{l}120 \\
(5.73 \%)\end{array}$ \\
\hline $\begin{array}{l}\text { Poor \& BPL card holder } \\
\text { benefitted }\end{array}$ & $944(8.27)$ & $370(19.68 \%)$ & $53(2.22 \%)$ & $229(9.59 \%)$ & $61(2.3 \%)$ & $\begin{array}{l}231 \\
(11.04 \%)\end{array}$ \\
\hline $\begin{array}{l}\text { Prosperous but BPL card } \\
\text { holder }\end{array}$ & $612(5.36 \%)$ & $8(0.43 \%)$ & $39(1.63 \%)$ & $55(2.3 \%)$ & $84(3.17 \%)$ & $\begin{array}{l}426 \\
(20.35 \%)\end{array}$ \\
\hline APL & $8(0.07 \%)$ & 0 & $3(0.12 \%)$ & $1(0.04 \%)$ & $4(0.15 \%)$ & 0 \\
\hline Total & 11416 & 1880 & 2386 & 2408 & 2650 & 2092 \\
\hline
\end{tabular}

\section{Other determinants of school education}

Table 5 shows that majority (87.39\%) of boys study in government or aided school and their study in Navodaya is negligible. Similarly most $(88.3 \%)$ of the girls study in government or aided school and their study in Navodaya and Kasturba Gandhi schools is negligible. Although government has made 
special provisions for ST to get admission in these schools but it is not getting momentum due to their lack of awareness, lack of interest and poor educational status of parents.

About $3.5 \%$ of girls and $5.17 \%$ of boys have to travel more than $2 \mathrm{~km}$ to reach their schools; most of the Sonbhadra boys and girls $(76.56 \%$ and $83.32 \%)$ have to travel to $1-2 \mathrm{~km}$ to reach their schools. Most of the children of ST families go on foot (92.2\%) to study in schools located within $2 \mathrm{~km}$ of their homes; use of vehicle is almost negligible. Sonbhadra children have no bicycles or other modes to reach their schools located $2 \mathrm{~km}$ or more; which is discouraging in difficult geographical terrain. About $80.13 \%$ of them feel to have less time available for study.

About $98.97 \%$ families perceive contribution of parent in study of their ward satisfactory. Most of the parents send their ward for study except remaining $0.41 \%$ for mid-day meal served in schools. As perceived by parents, school attendance of their ward is satisfactory in $92.05 \%$ cases, satisfactory academic achievement satisfactory in $91.97 \%$ cases, however facilities of electricity, fan, space and finance in term of scholarship is less than their expectation in school in $79.16 \%$ cases.

Education of neighbours of 76.5\% family is High School and Intermediate followed by $12.64 \%$ family having Primary and Middle pass neighbours and equal numbers (5.61\% and 5.42\%) of family having either illiterate or highly qualified neighbours. Sonbhadra families are having comparatively more $(15.95 \%)$ illiterate neighbours as well as no highly qualified neighbours.

Table 5: Status of other determinants of school education

\begin{tabular}{|c|c|c|c|c|c|c|c|}
\hline \multirow{2}{*}{\multicolumn{2}{|c|}{$\begin{array}{l}\text { Educational facility } \\
\text { parameters }\end{array}$}} & \multirow{2}{*}{$\begin{array}{l}\text { Number }(\%) \\
\text { Sonbhadra }\end{array}$} & \multicolumn{5}{|c|}{ District wise distribution: number (\%) } \\
\hline & & & Varanasi & Ghazipur & Deoria & Ballia & \\
\hline \multicolumn{2}{|c|}{ Type of school } & \multicolumn{6}{|c|}{ Number of children } \\
\hline \multirow[t]{4}{*}{$\begin{array}{l}\text { For } \\
\text { boys }\end{array}$} & Govt. & $8107(87.39 \%)$ & $\begin{array}{l}1502 \\
(97.79 \%)\end{array}$ & $\begin{array}{l}1800 \\
(90.22 \%)\end{array}$ & $\begin{array}{l}1433 \\
(74.6 \%)\end{array}$ & $\begin{array}{l}1928 \\
(88.04 \%)\end{array}$ & $\begin{array}{l}1444 \\
(88.32 \%)\end{array}$ \\
\hline & Private & $\begin{array}{l}1091 \\
(11.176 \%)\end{array}$ & $34(2.21 \%)$ & $193(9.67 \%)$ & $\begin{array}{l}412 \\
(21.48 \%)\end{array}$ & $\begin{array}{l}261 \\
(11.92 \%)\end{array}$ & $\begin{array}{l}191 \\
(11.68 \%)\end{array}$ \\
\hline & Navodaya & $79(0.85 \%)$ & 0 & $2(0.1 \%)$ & $76(3.96 \%)$ & $1(0.04 \%)$ & 0 \\
\hline & Total & 9277 & 1536 & 1995 & 1921 & 2190 & 1635 \\
\hline \multirow[t]{5}{*}{$\begin{array}{l}\text { For } \\
\text { girls }\end{array}$} & Govt. & $7338(88.3 \%)$ & $\begin{array}{l}1470 \\
(99.19 \%)\end{array}$ & $1509(79 \%)$ & $\begin{array}{l}1304 \\
(77.11 \%)\end{array}$ & $\begin{array}{l}1786 \\
(96.28 \%)\end{array}$ & $\begin{array}{l}1269 \\
(92.36 \%)\end{array}$ \\
\hline & Private & $841(10.12 \%)$ & $12(0.81 \%)$ & $\begin{array}{l}357 \\
(18.71 \%)\end{array}$ & $\begin{array}{l}300 \\
(17.74 \%)\end{array}$ & $\begin{array}{l}67 \\
(3.61 \%))\end{array}$ & $\begin{array}{l}105 \\
(7.64 \%)\end{array}$ \\
\hline & Navodaya & $117(1.41 \%)$ & 0 & $42(2.20 \%)$ & $75(0.43 \%)$ & 0 & 0 \\
\hline & Kasturba Gandhi & $14(0.17 \%)$ & 0 & 0 & $12(0.71 \%)$ & $2(0.11 \%)$ & 0 \\
\hline & Total & 8310 & 1482 & 1908 & 1691 & 1855 & 1374 \\
\hline
\end{tabular}




\begin{tabular}{|c|c|c|c|c|c|c|c|}
\hline \multicolumn{8}{|c|}{ Distance of school } \\
\hline \multirow[t]{4}{*}{$\begin{array}{l}\text { For } \\
\text { boys }\end{array}$} & Less than $1 \mathrm{~km}$ & $4826(52.02 \%)$ & $296(19.27 \%)$ & $\begin{array}{l}1006 \\
(50.42 \%)\end{array}$ & $\begin{array}{l}1464 \\
(76.21 \%)\end{array}$ & $\begin{array}{l}1675 \\
(76.48 \%)\end{array}$ & $\begin{array}{l}385 \\
(23.55 \%)\end{array}$ \\
\hline & $1-2 \mathrm{~km}$ & $3971(42.8 \%)$ & $\begin{array}{l}1222 \\
(79.56 \%)\end{array}$ & $\begin{array}{l}871 \\
(43.66 \%)\end{array}$ & $\begin{array}{l}360 \\
(18.74 \%)\end{array}$ & $\begin{array}{l}435 \\
(19.86 \%)\end{array}$ & $\begin{array}{l}1083 \\
(66.24 \%)\end{array}$ \\
\hline & More than $2 \mathrm{~km}$ & $480(5.17 \%)$ & $18(1.17 \%)$ & $118(5.91 \%)$ & $97(5.05 \%)$ & $80(3.65 \%)$ & $\begin{array}{l}167 \\
(10.21 \%)\end{array}$ \\
\hline & Total & 9277 & 1536 & 1995 & 1921 & 2190 & 1635 \\
\hline \multirow[t]{4}{*}{$\begin{array}{l}\text { For } \\
\text { girls }\end{array}$} & Less than $1 \mathrm{~km}$ & $4887(58.81 \%)$ & $260(17.54 \%)$ & $\begin{array}{l}1270 \\
(66.56 \%)\end{array}$ & $\begin{array}{l}1410 \\
(83.38 \%)\end{array}$ & $\begin{array}{l}1529 \\
(82.42 \%)\end{array}$ & $\begin{array}{l}418 \\
(30.42 \%)\end{array}$ \\
\hline & $1-2 \mathrm{~km}$ & $3132(37.69 \%)$ & $\begin{array}{l}1220 \\
(82.32 \%)\end{array}$ & $\begin{array}{l}569 \\
(29.82 \%)\end{array}$ & $\begin{array}{l}204 \\
(12.06 \%)\end{array}$ & $\begin{array}{l}263 \\
(14.18 \%)\end{array}$ & $\begin{array}{l}876 \\
(63.75 \%)\end{array}$ \\
\hline & More than $2 \mathrm{~km}$ & $291(3.5 \%)$ & $2(0.13 \%)$ & $69(3.61 \%)$ & $77(4.55 \%)$ & $63(3.4 \%)$ & $80(5.82 \%)$ \\
\hline & Total & 8310 & 1482 & 1908 & 1691 & 1855 & 1374 \\
\hline \multicolumn{2}{|c|}{ Total (boys \& girls) } & 17624 & 3028 & 3931 & 3671 & 4037 & 2957 \\
\hline \multicolumn{2}{|c|}{ Mode of transport } & \multicolumn{6}{|c|}{ Number of families sending their children to school } \\
\hline \multicolumn{2}{|c|}{ On foot } & $9319(92.2 \%)$ & $\begin{array}{l}1586 \\
(98.88 \%)\end{array}$ & $\begin{array}{l}1887 \\
(87.68 \%)\end{array}$ & $\begin{array}{l}1938 \\
(90.6 \%)\end{array}$ & $\begin{array}{l}2175 \\
(90.97 \%)\end{array}$ & $\begin{array}{l}1733 \\
(95.17 \%)\end{array}$ \\
\hline \multicolumn{2}{|c|}{ Bicycle } & $509(5.04 \%)$ & $16(0.1 \%)$ & $209(9.71 \%)$ & $99(4.63 \%)$ & $\begin{array}{l}114 \\
(4.77 \%)\end{array}$ & $71(3.9 \%)$ \\
\hline \multicolumn{2}{|c|}{ Cycle rickshaw } & $79(0.78 \%)$ & $1(0.62 \%)$ & $17(0.79 \%)$ & $42(1.96 \%)$ & $16(0.67 \%)$ & $3(0.16 \%)$ \\
\hline \multicolumn{2}{|c|}{ Auto rickshaw } & $132(1.31 \%)$ & $1(0.62 \%)$ & $31(1.44 \%)$ & $34(1.59 \%)$ & $61(2.55 \%)$ & $5(0.27 \%)$ \\
\hline \multicolumn{2}{|c|}{ Motor cycle } & $52(0.51 \%)$ & 0 & $4(0.18 \%)$ & $19(0.89 \%)$ & $20(0.84 \%)$ & $9(0.49 \%)$ \\
\hline \multicolumn{2}{|l|}{ Bus } & $16(0.16 \%)$ & 0 & $4(0.18 \%)$ & $7(0.33 \%)$ & $5(0.21 \%)$ & 0 \\
\hline \multicolumn{2}{|l|}{ Total } & 10107 & 1604 & 2152 & 2139 & 2391 & 1821 \\
\hline \multicolumn{8}{|c|}{ Average time to reach school } \\
\hline \multicolumn{2}{|c|}{ Normal } & $9121(90.24 \%)$ & $\begin{array}{l}1549 \\
(96.57 \%)\end{array}$ & $\begin{array}{l}1754 \\
(81.50 \%)\end{array}$ & $\begin{array}{l}1901 \\
(88.87 \%)\end{array}$ & $\begin{array}{l}2322 \\
(97.11 \%)\end{array}$ & $\begin{array}{l}1795 \\
(98.57 \%)\end{array}$ \\
\hline \multicolumn{2}{|l|}{ More } & $656(6.49 \%)$ & $24(1.5 \%)$ & $\begin{array}{l}370 \\
(17.19 \%)\end{array}$ & $206(9.63 \%)$ & $35(1.46 \%)$ & $21(1.15 \%)$ \\
\hline \multicolumn{2}{|c|}{ Excess } & $130(1.28 \%)$ & $31(1.93 \%)$ & $28(1.3 \%)$ & $32(1.5 \%)$ & $34(1.42 \%)$ & $5(0.27 \%)$ \\
\hline \multicolumn{8}{|c|}{ Availability of time for study } \\
\hline \multicolumn{2}{|l|}{ Less } & $8353(81.83 \%)$ & $\begin{array}{l}1313 \\
(81.85 \%)\end{array}$ & $\begin{array}{l}1151 \\
(53.48 \%)\end{array}$ & $\begin{array}{l}1987 \\
(92.89 \%)\end{array}$ & $\begin{array}{l}2263 \\
(94.64 \%)\end{array}$ & $\begin{array}{l}1639 \\
(90 \%)\end{array}$ \\
\hline \multicolumn{2}{|c|}{ Normal } & $1754(17.18 \%)$ & $\begin{array}{l}291 \\
(18.14 \% 0\end{array}$ & $\begin{array}{l}1001 \\
(46.51 \%)\end{array}$ & $152(7.1 \%)$ & $\begin{array}{l}128 \\
(5.35 \%)\end{array}$ & $182(10 \%)$ \\
\hline \multicolumn{8}{|c|}{ Contribution of parent in study } \\
\hline \multicolumn{2}{|c|}{ Satisfactory } & $\begin{array}{l}10003 \\
(98.97 \%)\end{array}$ & $\begin{array}{l}1582 \\
(98.63 \%)\end{array}$ & $\begin{array}{l}2125 \\
(98.75 \%)\end{array}$ & $\begin{array}{l}2113 \\
(98.78 \%)\end{array}$ & $\begin{array}{l}2378 \\
(99.45 \%)\end{array}$ & $\begin{array}{l}1805 \\
(99.12 \%)\end{array}$ \\
\hline \multicolumn{2}{|c|}{ Unsatisfactory } & $104(1.03 \%)$ & $22(1.37 \%)$ & $27(1.25 \%)$ & $26(1.22 \%)$ & $13(0.54 \%)$ & $16(0.88 \%)$ \\
\hline
\end{tabular}




\begin{tabular}{|c|c|c|c|c|c|c|}
\hline \multicolumn{7}{|c|}{ Parental attitude to school sending } \\
\hline For education & $9991(99.84 \%)$ & $\begin{array}{l}1599 \\
(99.69 \%)\end{array}$ & $\begin{array}{l}2122 \\
(98.6 \%)\end{array}$ & $\begin{array}{l}2090 \\
(97.7 \%)\end{array}$ & $\begin{array}{l}2381 \\
(99.58 \%)\end{array}$ & $\begin{array}{l}1799 \\
(98.79 \%)\end{array}$ \\
\hline For mid-day meal & $41(0.41 \%)$ & $2(0.12 \%)$ & $13(0.6 \%)$ & $8(0.37 \%)$ & $5(0.21 \%)$ & $13(0.71 \%)$ \\
\hline For both & $75(0.74)$ & $3(0.18 \%)$ & $17(0.79 \%)$ & $41(1.92 \%)$ & $5(0.21 \%)$ & $9(0.49 \%)$ \\
\hline \multicolumn{7}{|l|}{ School attendance } \\
\hline Satisfactory & $9303(92.05 \%)$ & $\begin{array}{l}1382 \\
(86.16 \%)\end{array}$ & $\begin{array}{l}1964 \\
(91.26 \%)\end{array}$ & $\begin{array}{l}2010 \\
(93.97 \%)\end{array}$ & $\begin{array}{l}2248 \\
(94.02 \%)\end{array}$ & $\begin{array}{l}1699 \\
(93.3 \%)\end{array}$ \\
\hline Unsatisfactory & $804(7.95 \%)$ & $222(13.84 \%)$ & $188(8.74 \%)$ & $129(6.03 \%)$ & $\begin{array}{l}143 \\
(5.98 \%)\end{array}$ & $122(6.7 \%)$ \\
\hline \multicolumn{7}{|l|}{ Facility in school } \\
\hline Less & $8001(79.16 \%)$ & $\begin{array}{l}1150 \\
(71.69 \%)\end{array}$ & $\begin{array}{l}1405 \\
(65.29 \%)\end{array}$ & $\begin{array}{l}1760 \\
(82.28 \%)\end{array}$ & $\begin{array}{l}2277 \\
(95.23 \%)\end{array}$ & $\begin{array}{l}1409 \\
(77.37 \%)\end{array}$ \\
\hline Good & $1632(16.15 \%)$ & $422(26.3 \%)$ & $\begin{array}{l}613 \\
(28.48 \%)\end{array}$ & $\begin{array}{l}305 \\
(14.25 \%)\end{array}$ & $30(1.25 \%)$ & $\begin{array}{l}262 \\
(14.38 \%)\end{array}$ \\
\hline Very good & $474(4.69 \%)$ & $32(1.99 \%)$ & $134(6.22 \%)$ & $74(3.46 \%)$ & $84(3.51 \%)$ & $\begin{array}{l}150 \\
(8.24 \%)\end{array}$ \\
\hline \multicolumn{7}{|l|}{ Study achievement } \\
\hline Satisfactory & $9296(91.97 \%)$ & $\begin{array}{l}1380 \\
(86.03 \%)\end{array}$ & $\begin{array}{l}2003 \\
(93.08 \%)\end{array}$ & $\begin{array}{l}1976 \\
(92.38 \%)\end{array}$ & $\begin{array}{l}2238 \\
(93.6 \%)\end{array}$ & $\begin{array}{l}1699 \\
(93.3 \%)\end{array}$ \\
\hline Unsatisfactory & $811(8.02 \%)$ & $224(13.96 \%)$ & $149(6.92 \%)$ & $163(7.62 \%)$ & $153(6.4 \%)$ & $122(6.7 \%)$ \\
\hline Total & 10107 & 1604 & 2152 & 2139 & 2391 & 1821 \\
\hline $\begin{array}{l}\text { Educational status of } \\
\text { neighbours }\end{array}$ & \multicolumn{6}{|c|}{ Number of families } \\
\hline Illiterate & $641(5.61 \%)$ & $300(15.95 \%)$ & $122(5.11 \%)$ & $62(2.57 \%)$ & $51(1.92 \%)$ & $\begin{array}{l}106 \\
(5.07 \%)\end{array}$ \\
\hline Primary, Middle & $1423(12.64 \%)$ & $194(10.32 \%)$ & $\begin{array}{l}488 \\
(20.45 \%)\end{array}$ & $\begin{array}{l}341 \\
(14.16 \% 0\end{array}$ & $\begin{array}{l}209 \\
(7.89 \%)\end{array}$ & $\begin{array}{l}191 \\
(9.13 \%)\end{array}$ \\
\hline $\begin{array}{l}\text { High School, } \\
\text { Intermediate }\end{array}$ & $8733(76.5 \%)$ & $\begin{array}{l}1386 \\
(73.72 \%)\end{array}$ & $\begin{array}{l}1619 \\
(67.85 \%)\end{array}$ & $\begin{array}{l}1624 \\
(67.44 \%)\end{array}$ & $\begin{array}{l}2377 \\
(89.7 \%)\end{array}$ & $\begin{array}{l}1727 \\
(82.55 \%)\end{array}$ \\
\hline \multirow[t]{2}{*}{ Highly educated } & $619(5.42 \%)$ & 0 & $157(6.58 \%)$ & $\begin{array}{l}381 \\
(15.82 \%)\end{array}$ & $13(0.49 \%)$ & $68(3.25 \%)$ \\
\hline & 11416 & 1880 & 2386 & 2408 & 2650 & 2092 \\
\hline Total & 11416 & 1880 & 2386 & 2408 & 2650 & 2092 \\
\hline
\end{tabular}

\section{Personal hygiene profiles of ST families}

Table 6 presents analysis of different components of personal hygiene practices such as hand wash practice, dental care, bathing, cleanliness of scalp hair and hand nails, and clothing. It reveals that hand wash after defecation, before and after meal is done by all families, however use of soap is not always there and ash is used when soap is not available. For dental care, maximum numbers of ST families 
either apply tooth powder by fingers $(41.65 \%)$ or use datoon (40.96\%). Use of toothbrush with dental paste is limited to $16.96 \%$ of families, they is use tobacco mixed powder locally known as gool or ash when toothpaste is not available. Most of them bath daily by occasional use of soap (44.54\%) or daily by soap (29.29\%), $15.47 \%$ of them bath daily without soap. Similarly, most of them clean their scalp hairs daily by occasional use of soap (54.54\%) or daily by soap (24.07\%). They use shampoo for cleaning of their scalp hairs occasionally (13.44\%) or bi-weekly (7.44\%), women cleans it preferably on special clay using multani mitti at place of soap for cleaning their long scalp hairs. Bath and hair cleaning becomes occasional once or twice a week during intense winter season in almost half of families especially among elderly men and children, and more so over without use of soap leading to emerging foul body smell enhanced more by unwashed shawl or blankets in front of bonfire locally known as alav. Maximum numbers of families (95.56\%) have cut and clean hand nails and $74.44 \%$ regularly cut and clean them, only $77.67 \%$ of ST families wear clean clothes.

Table 6: Personal hygiene status of ST families

\begin{tabular}{|c|c|c|c|c|c|c|c|}
\hline \multirow{2}{*}{\multicolumn{2}{|c|}{$\begin{array}{l}\text { Personal hygiene parameters } \\
\text { Sonbhadra }\end{array}$}} & \multirow{2}{*}{$\begin{array}{l}\text { Number of } \\
\text { families }(\%) \\
\text { Varanasi }\end{array}$} & \multicolumn{5}{|c|}{ District wise distribution: number (\%) } \\
\hline & & & \multirow[t]{2}{*}{ Ghazipur } & \multirow[t]{2}{*}{ Deoria } & \multirow[t]{2}{*}{ Ballia } & & \\
\hline \multicolumn{5}{|l|}{ Hand wash } & & & \\
\hline \multirow[t]{2}{*}{$\begin{array}{l}\text { After defecation } \\
\text { No }\end{array}$} & Yes & $11416(100 \%)$ & $\begin{array}{l}1880 \\
(100 \%)\end{array}$ & $\begin{array}{l}2386 \\
(100 \%)\end{array}$ & $\begin{array}{l}2408 \\
(100 \%)\end{array}$ & $\begin{array}{l}2650 \\
(100 \%)\end{array}$ & $\begin{array}{l}2092 \\
(100 \%)\end{array}$ \\
\hline & 0 & 0 & 0 & 0 & 0 & 0 & \\
\hline \multirow[t]{2}{*}{ Before meal No } & Yes & $11416(100 \%)$ & $\begin{array}{l}1880 \\
(100 \%)\end{array}$ & $\begin{array}{l}2386 \\
(100 \%)\end{array}$ & $\begin{array}{l}2408 \\
(100 \%)\end{array}$ & $\begin{array}{l}2650 \\
(100 \%)\end{array}$ & $\begin{array}{l}2092 \\
(100 \%)\end{array}$ \\
\hline & 0 & 0 & 0 & 0 & 0 & 0 & \\
\hline \multirow[t]{2}{*}{ After meal No } & Yes & $11416(100 \%)$ & $\begin{array}{l}1880 \\
(100 \%)\end{array}$ & $\begin{array}{l}2386 \\
(100 \%)\end{array}$ & $\begin{array}{l}2408 \\
(100 \%)\end{array}$ & $\begin{array}{l}2650 \\
(100 \%)\end{array}$ & $\begin{array}{l}2092 \\
(100 \%)\end{array}$ \\
\hline & 0 & 0 & 0 & 0 & 0 & 0 & \\
\hline \multicolumn{8}{|l|}{ Dental care } \\
\hline \multicolumn{2}{|c|}{ Tooth brushes with dental paste } & $\begin{array}{l}1937 \\
(16.96 \%)\end{array}$ & $144(7.66 \%)$ & $\begin{array}{l}375 \\
(15.71 \%)\end{array}$ & $\begin{array}{l}607 \\
(25.2 \%)\end{array}$ & $\begin{array}{l}370 \\
(13.96 \%)\end{array}$ & $\begin{array}{l}441 \\
(21.08 \%)\end{array}$ \\
\hline \multicolumn{2}{|c|}{$\begin{array}{l}\text { Applying tooth powder by } \\
\text { fingers }\end{array}$} & $\begin{array}{l}4755 \\
(41.65 \%)\end{array}$ & $\begin{array}{l}1118 \\
(59.47 \%)\end{array}$ & $\begin{array}{l}1276 \\
(53.48 \%)\end{array}$ & $\begin{array}{l}603 \\
(25.04 \%)\end{array}$ & $\begin{array}{l}918 \\
(34.64 \%)\end{array}$ & $\begin{array}{l}840 \\
(40.15 \%)\end{array}$ \\
\hline \multicolumn{2}{|l|}{ By datoon } & $\begin{array}{l}4676 \\
(40.96 \%)\end{array}$ & $\begin{array}{l}610 \\
(32.44 \%)\end{array}$ & $\begin{array}{l}722 \\
(30.26 \%)\end{array}$ & $\begin{array}{l}1182 \\
(49.08 \%)\end{array}$ & $\begin{array}{l}1355 \\
(51.13 \%)\end{array}$ & $\begin{array}{l}807 \\
(38.57 \%)\end{array}$ \\
\hline \multicolumn{2}{|c|}{ Only by water gargle } & $48(0.35 \%)$ & $8(0.42 \%)$ & $13(1.8 \%)$ & $\begin{array}{l}16 \\
(43.44 \%)\end{array}$ & $7(0.26 \%)$ & $4(0.19 \%)$ \\
\hline \multicolumn{8}{|l|}{ Bathing practice } \\
\hline \multicolumn{2}{|c|}{ Daily only by water } & $\begin{array}{l}1767 \\
(15.47 \%)\end{array}$ & $\begin{array}{l}638 \\
(33.94 \%)\end{array}$ & $\begin{array}{l}280 \\
(11.73 \%)\end{array}$ & $\begin{array}{l}315 \\
(13.08 \%)\end{array}$ & $\begin{array}{l}301 \\
(11.36 \%)\end{array}$ & $\begin{array}{l}233 \\
(11.14 \%)\end{array}$ \\
\hline \multicolumn{2}{|l|}{ Daily by soap } & $\begin{array}{l}6305 \\
(55.23 \%)\end{array}$ & $\begin{array}{l}580 \\
(30.85 \%)\end{array}$ & $\begin{array}{l}1171 \\
(49.1 \%)\end{array}$ & $\begin{array}{l}1094 \\
(45.43 \%)\end{array}$ & $\begin{array}{l}1828 \\
(68.98 \%)\end{array}$ & $\begin{array}{l}1632 \\
(78.01 \%)\end{array}$ \\
\hline
\end{tabular}




\begin{tabular}{|c|c|c|c|c|c|c|c|}
\hline \multicolumn{2}{|c|}{ Occasionally by soap } & $\begin{array}{l}3344 \\
(29.29 \%)\end{array}$ & $\begin{array}{l}662 \\
(35.21 \%)\end{array}$ & $\begin{array}{l}935 \\
(39.18 \%)\end{array}$ & $\begin{array}{l}999 \\
(41.49 \%)\end{array}$ & $\begin{array}{l}521 \\
(19.66 \%)\end{array}$ & $\begin{array}{l}227 \\
(10.85 \%)\end{array}$ \\
\hline \multicolumn{8}{|c|}{ Cleanliness of scalp hair } \\
\hline \multicolumn{2}{|c|}{ Daily only by water } & $\begin{array}{l}1244 \\
(10.89 \%)\end{array}$ & $\begin{array}{l}395 \\
(21.01 \%)\end{array}$ & $\begin{array}{l}198 \\
(8.29 \%)\end{array}$ & $\begin{array}{l}201 \\
(8.34 \%)\end{array}$ & $\begin{array}{l}192 \\
(7.24 \%)\end{array}$ & $\begin{array}{l}258 \\
(12.33 \%)\end{array}$ \\
\hline \multicolumn{2}{|c|}{ Daily by soap } & $\begin{array}{l}2748 \\
(24.07 \%)\end{array}$ & $\begin{array}{l}300 \\
(15.95 \%)\end{array}$ & $\begin{array}{l}457 \\
(19.15 \%)\end{array}$ & $\begin{array}{l}609 \\
(25.29 \%)\end{array}$ & $\begin{array}{l}900 \\
(33.96 \%)\end{array}$ & $\begin{array}{l}482 \\
(23.04 \%)\end{array}$ \\
\hline \multicolumn{2}{|c|}{ Occasionally by soap } & $\begin{array}{l}5085 \\
(44.54 \%)\end{array}$ & $\begin{array}{l}958 \\
(50.75 \%)\end{array}$ & $\begin{array}{l}1183 \\
(49.58 \%)\end{array}$ & $\begin{array}{l}1203 \\
(49.95 \%)\end{array}$ & $\begin{array}{l}1155 \\
(43.58 \%)\end{array}$ & $\begin{array}{l}586 \\
(28.01 \%)\end{array}$ \\
\hline \multicolumn{2}{|c|}{ Occasionally by shampoo } & $\begin{array}{l}1489 \\
(13.04 \%)\end{array}$ & $\begin{array}{l}211 \\
(11.22 \%)\end{array}$ & $\begin{array}{l}248 \\
(10.39 \%)\end{array}$ & $\begin{array}{l}309 \\
(12.83 \%)\end{array}$ & $\begin{array}{l}214 \\
(8.07 \%)\end{array}$ & $\begin{array}{l}507 \\
(24.23 \%)\end{array}$ \\
\hline \multicolumn{2}{|c|}{ Bi-weekly by shampoo } & $850(7.44 \%)$ & $16(0.85 \%)$ & $\begin{array}{l}300 \\
(12.57 \%)\end{array}$ & $86(3.57 \%)$ & $\begin{array}{l}189 \\
(7.13 \%)\end{array}$ & $\begin{array}{l}259 \\
(12.38 \%)\end{array}$ \\
\hline \multicolumn{2}{|c|}{ Never by shampoo } & 0 & 0 & 0 & 0 & 0 & 0 \\
\hline \multicolumn{8}{|c|}{ Cleanliness of hand nails } \\
\hline \multirow[t]{2}{*}{ Status } & Cut \& clean & $\begin{array}{l}10908 \\
(95.56 \%)\end{array}$ & $\begin{array}{l}1834 \\
(97.55 \%)\end{array}$ & $\begin{array}{l}2238 \\
(93.8 \%)\end{array}$ & $\begin{array}{l}2215 \\
(91.99 \%)\end{array}$ & $\begin{array}{l}2644 \\
(99.77 \%)\end{array}$ & $\begin{array}{l}1977 \\
(94.5 \%)\end{array}$ \\
\hline & Long dirty & $508(4.44 \%)$ & $46(2.44 \%)$ & $148(6.2 \%)$ & $\begin{array}{l}193 \\
(8.01 \%)\end{array}$ & $6(0.23 \%)$ & $\begin{array}{l}115 \\
(5.5 \%)\end{array}$ \\
\hline \multirow{2}{*}{$\begin{array}{l}\text { Practice } \\
\text { of regular } \\
\text { cutting }\end{array}$} & Yes & $\begin{array}{l}8498 \\
(74.44 \%)\end{array}$ & $\begin{array}{l}1326 \\
(70.53 \%)\end{array}$ & $\begin{array}{l}1669 \\
(69.95 \%)\end{array}$ & $\begin{array}{l}1790 \\
(74.33 \%)\end{array}$ & $\begin{array}{l}1991 \\
(75.13 \%)\end{array}$ & $\begin{array}{l}1722 \\
(82.31 \%)\end{array}$ \\
\hline & No & $\begin{array}{l}2918 \\
(25.56 \%)\end{array}$ & $\begin{array}{l}554 \\
(29.47 \%)\end{array}$ & $\begin{array}{l}717 \\
(30.05 \%)\end{array}$ & $\begin{array}{l}618 \\
(25.66 \%)\end{array}$ & $\begin{array}{l}659 \\
(24.87 \%)\end{array}$ & $\begin{array}{l}370 \\
(17.68 \%)\end{array}$ \\
\hline \multicolumn{8}{|l|}{ Clothing } \\
\hline \multicolumn{2}{|l|}{ Neat clean } & $664(5.81 \%)$ & $98(5.21 \%)$ & $\begin{array}{l}169 \\
(7.08 \%)\end{array}$ & $\begin{array}{l}133 \\
(5.52 \%)\end{array}$ & $\begin{array}{l}152 \\
(5.73 \%)\end{array}$ & $\begin{array}{l}112 \\
(5.35 \%)\end{array}$ \\
\hline \multicolumn{2}{|c|}{ Satisfactory cleanliness } & $\begin{array}{l}8203 \\
(71.86 \%)\end{array}$ & $\begin{array}{l}1271 \\
(67.6 \%)\end{array}$ & $\begin{array}{l}1613 \\
(67.6 \%)\end{array}$ & $\begin{array}{l}1670 \\
(69.35 \%)\end{array}$ & $\begin{array}{l}1890 \\
(71.32 \%)\end{array}$ & $\begin{array}{l}1759 \\
(84.08 \%)\end{array}$ \\
\hline \multicolumn{2}{|l|}{ Dirty } & $\begin{array}{l}2549 \\
(22.33 \%)\end{array}$ & $\begin{array}{l}511 \\
(27.18 \%)\end{array}$ & $\begin{array}{l}604 \\
(25.31 \%)\end{array}$ & $\begin{array}{l}605 \\
(25.12 \%)\end{array}$ & $\begin{array}{l}608 \\
(22.94 \%)\end{array}$ & $\begin{array}{l}221 \\
(10.56 \%)\end{array}$ \\
\hline \multicolumn{2}{|l|}{ Total } & 11416 & 1880 & 2386 & 2408 & 2650 & 2092 \\
\hline
\end{tabular}

\section{DISCUSSION}

\section{Status of education}

\section{Education of husband or wife in ST family}

Maximum numbers (55.9\%) of husband or wife in ST families are primary to $10+2$ educated followed by illiteracy (31.4\%) as presented in table 1.2 . Only $4.9 \%$ husbands or wives in families are graduate or more educated. Illiteracy is a major educational problem of the tribes. Finding in present study are in 
conformity with the NSSO, 1991 report that literacy among the tribes is very low (25.9\%) and specially so among the tribal females (14.5\%) and the level of education among the most of the scheduled tribes is only up to primary level. Basu (2000) also mentioned the lowest level of literacy (4.1\%) among females in Rajasthan as they have no faith in formal educational organization; they feel no urge to educate their children. Since most of the tribal are poor, education appears to be a luxury for them.

Although there is a significant increase in the literacy of population of all categories in India, the tribal are far behind from the national increase. These findings are comparable to those of different ST communities during census 2001. Among the five STs (Tharu, Buksa, Bhotia, Janusari and Raji) in U.P., only Janusari have shown the overall literacy rate (51.1\%) higher than that of national average (47.1\%). All the five STs have shown male \& female literacy rates $(48.4 \%$ and $20.7 \%)$ lower than that of national averages $(59.2 \% \& 34.8 \%)$ for all ST. Educational status declines sharply from secondary education onward. Present finding is also comparable to only 59\% among ST in 2011 against the literacy rate of overall population (73\%) in all social group and (66\%) in SC population. Government of India (2013) also reported it to be $55.7 \%$ among tribes of U.P. less than national average.

Educational achievement among tribes are still far behind as per trend of progress not only from general population but also from year 2001 to 2011 in specific tribes. Although literacy of STs have improved but its gap between male and female still not filled significantly. Many factors contribute to low level of literacy. Due to poor economic conditions most of them cannot afford to send their children to schools, those who do enter the school often drop out of school at early age on account of several factors or have to take care of young children in family and or to contribute to family income.

\section{Education of children}

Maximum number (42.3\%) of ST families are sending or have sent their all children to school followed by $39.2 \%$ families sending or have sent their $>50 \%$ children to school. On average $12.2 \%$ of families are still not sending any children to school. Sonbhadra ST families have poor records of education as high as $21.1 \%$ of them are not sending any children to school and as low as $3.2 \%$ of them are not sending their all children. Major concern is their children's presence in schools that too visible mainly during admission season to get the free bag and only at the time of mid-day meal distribution, fact noted in almost all study districts.

Present finding is in conformity with the observed school dropout rate reported by the government agencies. School dropout rate has been very high in ST. It was $35.6 \%$ in class I to V, $55 \%$ in class I to VIII and $70.9 \%$ in class I to $\times$ in 2010-11 and significantly higher than all categories (27, 40.6 and $49.3 \%$ respectively). Dropout rate reported from U.P. was 22.2 in class I to VIII (27.9 in boys and 13.9 in girls) in ST community; dropout rates are alarmingly higher in the higher classes.

\section{Socio-economic status}

\section{Socio-economic grades}

In present study, $73.4 \%$ of ST families are in poor grade as presented in table 1 . As per $68^{\text {th }}$ round 
report of NSSO (2011-12), 21.9 percent of overall national population is poor and U.P having 30-40\% population below poverty line, it is comparatively very high amongst ST in eastern U.P. Finding is in conformity with IHD survey report which stated that $49.6 \%$ of STs are poor in contrast to $12 \%$ of general group. It reaffirms the observation made by Gare (1983) that the extremely low levels of literacy, education, health and nutrition status, inadequate unemployment and unproductive agriculture of tribal put a constraint on improving their socio-economic status. Although government aims at raising the socioeconomic standard of tribal to the level of general population (Government of India 2013), it is not getting success in ground reality in eastern U.P.

In present study, only $51.5 \%$ of ST persons living in Ghazipur are poor compared to $97.3 \%$ of those living in Sonbhadra. Although it appears largely due to hilly terrain of Sonbhadra, it needs insight of other contributing factors. It is in conformity with the statement made by Aphale and Bairagi (1984) that high percentage of disparity among the tribal are due to their backwardness, ignorance and poverty and, in spite of the budget provisions, some schemes of Tribal Sub Plans were not implemented and funds of individual benefits were diverted.

Present finding also reaffirms the observation made by Belshaw (1972) that though a lot has been done for tribal's social and economic betterment, yet a great deal remains to be done. Singh (1997) also mentioned that the tribal in India is the most adversely affected ethnic group due to the development in post-independence India. Development should not be studied in isolation, as it is not synonymous of with the growth of few affluent persons. Amartya Sen (1999) further supplemented that unless the capabilities among human beings are adequately addressed and deprivations faced by marginalized groups are overcome, development cannot take place. Capabilities and human freedoms can only be achieved when the people are guaranteed political freedom, economic facilities, social opportunities, transparency, and security. Government's effort of tribal welfare with protective developmental measures has not yielded any remarkable impact on tribal development and the tribal development has been a challenge for government in the area of socio-economic aspect.

The data from NIRD Report (2012) presented that poverty among STs has declined sharply from 61.9\% in 2004-05 to 47.1\% in 2009-10 in rural areas and significantly in urban areas by Tendulkar Methodology. The high rural poverty can be attributed to lower farm incomes due to subsistence agriculture, lack of sustainable livelihoods in rural areas, impact of rise in prices of food products on rural incomes, lack of skills, and underemployment.

These finding reaffirms the observation made by Rai (2017) that their economic situation is worse than other communities in society, majority of them are deprived from the basic needs of life. Compared to urban areas, situation of tribes living in remote area is worse. Tribes are scattered politically in eastern U.P., having no effect on politics. Although welfare plans such as subsidizing housing like Lohia, Indira, Kashiram Awas Yojna exists for poor in rural area, but tribes are not being benefitted due to several reasons. Education level is very low and economic factors are biggest hurdle in getting higher education in tribes, they are victims of inequality, exploitation and oppression. 
Present study finding is in conformity with the conclusion arrived by Rai (2018) that tribes of U.P. are living in conditions of deprivation; their economic condition and standard of living are very low as most of them don't have land, asset and education. They are having dual status in the state because of Act 2002 as some of these ST are notified in SC and even backward caste in other districts of U.P. In the list of STs prepared in 1950, primitiveness and backwardness were the tests applied for specifying a Scheduled Tribe. Present study finding confirms that general backwardness in all respects is still rampant in ST living in eastern U.P.

\section{Livelihood profile of family}

ST families are primarily agrarian and most of them earn their livelihood from irregular labour (83.82\%), only $1.67 \%$ has sufficient and $0.73 \%$ insufficient farming and their economic status is low $(88.42 \%)$ as presented Table 2.2. It affirms the observation made by Aphale and Bairagi (1984) that agriculture is the most crucial occupation of the tribes but their yields are poor because they continue to cultivate land and practice forming in their traditional primitive manner. Gare (1983) reported that agriculture in the tribal areas is mostly unproductive and it is incapable of providing adequate employment. NSSO Survey report 2012 shows self-employment in agriculture is dominant source of income among ST households (37\%) as well as among all groups (31.9\%). Non-agriculture self-employment is lowest among ST (7\%) than that of all groups (15.5\%). Considerable proportion of ST population (46.5\%) is engaged in rural labour and $44 \%$ of them are self-employed. Jaiswal (2017) also found majority of them to be agriculture labourers.

Present study finding reveals that small shop, occupation, service contributes $2 \%$ earnings equally from insufficient and sufficient amounts. It is in conformity with the $5^{\text {th }}$ Economic census report (2005) that tribal worked maximum in retail sector followed by in manufacturing sector and significantly less in service sector in compare to other social groups. It is also comparable to the 2011 census finding that ST were employed as total workers $(58 \%)$, main workers $(37.6 \%)$, cultivators $(34.5 \%)$, agriculture labourers (44.5\%), house-hold industry (1.8\%), other works (19.2\%) and $42 \%$ of ST were non-workers. Finding is also in line with Government of India, 2013 that in the last decade, about 3.5 million tribal people are leaving agriculture and agriculture-related activities to enter the informal labour market.

In present study, majority of ST families are poor but $47.7 \%$ of them don't have BPL card, $36.79 \%$ possess BPL card but not benefitted by welfare schemes, $8.27 \%$ are card holders and get benefits and $1.81 \%$ possess card but suffer due to diseases and addiction. It also reaffirms the observation made by Singh (1997) that Government's efforts of tribal welfare with protective developmental measures has not yielded any remarkable impact on tribal development and the tribal development has been a challenge for government.

Prosperous people manage to get BPL card (5.36\%) which is lowest $(0.43 \%)$ in Sonbhadra. Misuse of benefits of welfare schemes further deprives the eligible families. Sonbhadra is worst affected due to its adverse geographical location and low educational status. 


\section{Other determinants of education}

Government has made special provision of Navodaya and Kasturba Gandhi schools for socially deprived classes of society especially for rural people; these schools provide quality education and personality development with free food and accommodation. School education in Navodaya and Kasturba Gandhi schools is negligible due to several factors such as lack of awareness, poor educational achievement of their wards unable to qualify entrance tests, lack of social support. Present finding is comparable to observation made by Pradhan (2011) who described that despite special initiatives like Ashram schools, introducing vernacular at primary level, and teaching in local dialects, the tribal are still lagging behind the non-tribal. It is in conformity with Burman (2009) who pointed out more number of Ashram schools should be opened in their areas, and the government rules are to be relaxed so that more and more children can get into the school system.

About $50-76 \%$ children have to travel to $1-2 \mathrm{~km}$ to reach their schools. It leads not only discouraging children for education but also less availability of time to study. Most of the children of ST families in all districts go on foot (92.2\%) to study in schools, $80.13 \%$ of them feel to have less time available for study. Non-availability of vehicles even bicycles in family is one of the important causes of difficulty in reaching schools especially in remote area. Present finding is in conformity with Burman (2009) who pointed out that even after the implementation of Sarva Siksha Abhiyan, only $88.46 \%$ of ST households are covered under primary schools in a radius of $1 \mathrm{~km}$.

Less availability of study time is also attributed to indiscipline, time wastage in play and sometimes in family liability, child labour, less motivation toward education, poor quality of locality including low educational status of their neighbours. Hasnain (2004) also reported that deprived family see children as economic assets to supplement the family income by working with the parents and with others due to poor economic condition and subsistence economy. In a situation of dire necessitates of life, education becomes a matter of luxury for the tribal family. It is further in conformity with Ambuselvi et al. (2018) that majority of the tribal children were engaged in crucial family work like cattle grazing, labour on work sites, collecting fire wood which deprive them to spare adequate time for study and also boost their school absenteeism tendency.

Although parents of $98.97 \%$ of families perceive their satisfactory contribution in study of their ward but ground reality may differ from this observation as academic achievement of tribe students reported by studies are on lower spectrum and it could have been better if really supported by their parents. Jayaswal et al. (2003) found that the parent of higher achievers exerted significantly more support to their children and parents of lower achievers were not strongly ambitious of children's upward mobility. Parents in most of the ST family in present study are lower achievers as maximum numbers $(55.9 \%)$ of husband or wife in ST families in all of the selected five districts are primary to 10+2 educated followed by illiteracy (31.4\%).

Although most of the parents tells to send their ward for study (except remaining $0.41 \%$ for mid-day meal served in schools) but satisfactory perception of parents toward their wards' achievement does not match with ground reality of academic outcome as most of these children are unable to do simple 
academic exercises as mentioned by ASER report (2014). Low educational status of their guardians makes them unable to assess their wards achievement. Poor education of neighbours is further deterrent in school education as almost half of their neighbours are illiterate.

As perceived by parents, facilities of electricity, fan, space and finance in term of scholarship is less than their expectation in school in $79.16 \%$ cases. It is in conformity with observation of Ambuselvi et al. (2018) who reported that residential schools are very poor in infrastructural facilities and have poor hygiene and poor noon meal program. Educational facilities like free textbook, free uniform, stipends are also poor. Twenty five percent of them revealed that the teachers in the tribal schools are frequently absent. It is further aggravated by the fact that lack of infrastructure facility, communication system, transport facilities make teachers' to hesitate to work with full fledge dedication in tribal areas. Lack of resources, poverty, imbalanced diet, climatic conditions, lack of medical facilities and myths of diseases and their treatments are some of the reasons for frequent illness among them keep away from regular study. Present observation is also in direction with Jha and Jhingan (2002) who mentioned that even where there is reasonable infrastructure and student enrolment, regular school attendance is a problem in the tribal areas, and forecasted that to make matters worse, the old problem of teacher absenteeism that in the tribal areas are known for to persist.

\section{Personal hygiene profile of family}

Finding of personal hygiene are comparable to observations made by Jaiswal (2017) that ST families in Sonbhadra keep their place mostly clean. Women are very active to clean their boundary, most of them take daily bath, which is generally avoided in winter season usually by old people and children, they use multani-mitti and sometime shampoo for hair cleaning especially by women, they use datoon sticks and sometimes tobacco powder and ash to rub the teeth for cleaning purpose. Personal hygiene status also affects to education. Poor personal hygiene practice such as not taking bath, wearing dirty clothes including not wearing school uniform due to lack of water facility, poor socio-economic condition of parents and their low awareness creates discomfort in school environment. Poor personal hygiene among children predisposes them to discrimination in school that leads to not only poor educational outcome but also comparatively higher dropout rate. Irregular school attendance further leads to deprivation from scholarship facility. Adults also realize similar discrimination in employment.

Human Rights Watch (2014) records that the Ghasiya tribal children of Sonbhadra were made to sit in a single grade irrespective of their ages, that also separately from the other students. The Principal of the concerned school stated that the tribal children were a 'big problem' in the school. Such prejudice not only precludes the potential for tribal children learning in the classroom, it also perpetuates discrimination and exclusion. The problem has become endemic. Personal hygiene of students has direct impact on teaching and learning due to several actors. These children don't want to go to school if their clothes are dirty. It happens once or twice a week, teachers say to them to sit in the back of class. 


\section{CONCLUSION AND RECOMMENDATIONS}

Most of the heads of ST families are either $10+2$ educated (55.9\%) or illiterate (31.4\%). Although their educational achievement from earlier primary pass level has improved in last two decades but persistence of low level of graduation or skilled education and higher illiteracy is a matter of concern. Such education status is depriving them from gateway for better livelihood in local society and national job market. High illiteracy of parents is not creating urge for better education in their children. Literacy gap between male and female still not filled significantly, which is detrimental for not only better educational outcome of future generation but also gender biased. On average $12.2 \%$ of families are still not sending their children to school which is as high as $21.1 \%$ in Sonbhadra, dismal records of child education along with persistent high dropout rate in ST families is also worrisome. School attendance mainly during admission season and only at the time of mid-meal distribution reflects the casual approach toward school education. Education level is very low and economic factors are biggest hurdle in getting higher education in tribes, they are victims of inequality, exploitation and oppression. All of these noted discrepancies needs corrective approach to improve the status of education in ST communities.

Most of the ST families belongs to either poor (73.4\%) or lower middle (24\%) socio-economic grade that is as high as $96.8 \%$ poor in Sonbhadra. Highest population density and lowest per capita agricultural production along with second lowest rank of industrial production in eastern U.P. has led to its economic backwardness. Most of them especially in Sonbhadra are deprived of the basic needs of life; high percentage of disparity among the tribal are due to their backwardness, ignorance and poverty. In spite of the special budget provisions, some relevant schemes not implemented even in high priority area of Sonbhadra, establishment of cement and other allied factories and giant thermal plants have not benefitted native tribes. Robust institutions are needed to bridge of wide gaps between ST and general population in rapidly changing socio-economic conditions. The low representation of tribes to the total population in U.P. often excludes from development processes hence their adequate political representation is required for their uplift and empowerment.

ST families are primarily agrarian and most of them earn their livelihood from irregular mostly agriculture labour $(83.82 \%$ ), only $2.4 \%$ has farming resulting poor yield of their small inadequate land with traditional manner of farming complicated by poor fertility of their land which let the tribal people fall the pray to indebtedness. Most of these tribes do not have land, asset and education; most of them are agricultural labourers and earns low farm incomes due to subsistence agriculture having more volatile and uncertain nature. Other contributing factors are lacks of knowledge, skill, production how to generate the income, use of modern technology, agriculture supported programme, agriculture market, not enough of development structure, and they are not managing their income. Although majority of them are poor but only $45 \%$ have BPL cards and some of these card holders are not getting its benefits due lack of education. Problem of livelihood of these tribes and exploitation can be resolved through education and self-employment. They need to be encouraged and confident to connect their traditional professions through self-employment schemes, which should be readily available locally within their reach. To enable acceptance of new technologies, training should be imparted in their own 
dialect/language for easy understanding. The discriminatory credit policy of the financial institutions is a major deterrent in the participation of STs in business. Entrepreneurs among STs must be given credit facility on par with other social groups. Further, all steps must be taken by the Government for skill development among the tribal youth to make them employable or self-employed, according to their aspirations.

Many factors contribute to low level of education. Due to poor economic conditions most of them cannot afford to send their children to schools, those who do enter the school often drop out of school at early age on account of several factors or have to take care of young children in family and or to contribute to family income. Apart from socio-economic factor, there are other determinants of education, which needs further strengthening. Study of ST children in residential school like Navodaya and Kasturba Gandhi schools providing quality education and personality development with free food and accommodation is negligible due to several factors such as lack of awareness, poor educational achievement of their wards unable to qualify entrance tests, lack of social support. Government's special initiatives like opening of residential Ashram schools, introducing vernacular at primary level, and teaching in local dialects is not getting desired success. Only half of children have schools within $1 \mathrm{~km}$ of their home and most of the Sonbhadra boys and girls $(76.56 \%$ and $83.32 \%$ ) have to travel to $1-2 \mathrm{~km}$ to reach their schools. Determination of Sarva Siksha Abhiyan to operate primary school within a radius of 1 $\mathrm{km}$ of population is lacking in remote and sparsely located ST family especially in Sonbhadra. Most of the children (92.2\%) go on foot to study in schools located within $2 \mathrm{~km}$ of their homes, only $5.04 \%$ use bicycles, Sonbhadra children almost lack bicycles or other modes to reach their schools located $2 \mathrm{~km}$ or more. Non-availability of vehicles even bicycles in family is one of the important causes of difficulty in reaching schools especially in remote area. Majority $(80.13 \%)$ of students feel to have less time available for study due to several identified reasons which needs to be addressed with more sincere effort to reduce the school dropout rate of ST students.

Parent of higher achievers exert significantly more support to their children but parents in most of the ST family in present study are lower achievers, low educational status of their guardians makes them unable to assess their wards achievement. There is a need for regular social audits to monitor the functioning of schools, monitoring committee at the block level for primary and middle schools and district level for high and higher secondary schools should be set up. In present study, about $30 \%$ of the neighbours are either illiterate or primary pass only, which present the poor educational environment for school going children, which is worse in Sonbhadra. Community involvement is needed for success of the educational institutions. Information on various schemes, benefits should be provided to not only create demand for scholarship including other entitlement and education among guardians but also motivate them to see education as gateway for better livelihood in local society and national job market.

Facilities of electricity, fan, space and financial support (in term of scholarship) is comparatively less (79.16\%) as per their expectation. Educational facilities (in term of availability of textbook, free uniform, stipends and mid-day meals) of ST are poorer in comparison to other population. Teachers frequently remain absent due to lack of infrastructure facility, communication system, and transport facility in tribal areas. Lack of resources, poverty, imbalanced diet, climatic conditions, lack of medical facilities 
and myths of diseases and their treatments are some of the reasons for frequent illness among tribal children, which keeps them away from regular study. Regular school attendance is a problem in the tribal areas in spite of reasonable infrastructure, which need more attention. Since the educational scenario in tribal areas is marked by poor infrastructure, providing adequate infrastructure, such as classrooms, teachers and teaching aids as well as basic facilities like electricity, water, boundary walls and toilets is essential for the proper functioning of these schools and from the perspective of security and safety of children. Keeping in view the difficulties of adjusting to a new cultural environment, teachers for schools in the tribal regions should be recruited locally.

Poor personal hygiene also affects education. Poor personal hygiene practice such as not taking bath, wearing dirty clothes including not wearing school uniform due to lack of water facility, poor socioeconomic condition of parents and their low awareness creates discomfort in school environment. Poor personal hygiene among children predisposes them to discrimination in school environment. Irregular school attendance further leads to deprivation from scholarship facility. Status of personal hygiene should be improved by appropriate motivation and counselling. Teachers must be trained to set good practices for social inclusion and equity, such as encouraging children from marginalized group to participate in school activities, ensuring more frequent collaboration between children of different social groups and, promoting innovative activities aimed at inclusion.

\section{REFERENCES}

1. Aphale, M.B. and Bairagi, K.V. 1984. Evaluation study of Integrated Tribal Development Project Thane-I, Jawahar, Tribal Research Bulletin, Published by TRTI, Pune.

2. Aggarwal O.P., Bhasin S.K., Sharma A.K., Chhabra P., Aggarwal K. and Rajoura O.P. 2005. A New Instrument (Scale) for measuring the Socioeconomic Status of a Family, Indian Journal of Community Medicine, 30(4).

3. Agriculture Census. 2011. Department of Agriculture and Farmers Welfare, Ministry of Agriculture and Farmers Welfare, New Delhi, retrieved from agcensus.nic.in/document/agcensus2010/allindia201011H.pdf

4. Anbuselvi, P., Leeson, P. John and Anbuselvi, G. 2018. Education of tribal children in India: A case study, International Journal of Advanced and Innovative Research, 24(3): 206.

5. Annual Status of Education Report (ASER), 2014. Retrieved from http://img.asercentre.org/docs/Publications/ ASER\%20Reports/ASER\%202014/fullaser2014mainreport_1.pdf

6. Basu, S. 2000. Dimensions of Tribal Health in India, Health and Population-Perspectives and Issues, 23(2): 61-70.

7. Belshaw, C.S. 1972. Development: The contribution of Anthropology, International Social Science Journal.

8. Burman, B.K. Roy. 2009. 'What Has Driven the Tribals of Central India to political Extremism Mainstream', XLVII (44), http://www.mainstreamweekly.net/ article1704.html.

9. Census 2011. Registrar General of India, Demographic Status of Scheduled Tribe Population in India, New Delhi.

10. Economic Census, 2005. Economic Census $5^{\text {th }}$ Report, Ministry of Statistics, Planning and Implementation, New Delhi, retrieved from www.mospi.gov.in/economic-census-3. 
11. Gare, G.M. 1983. Tribal development program, Tribal Research Bulletin, September, TRTI, Pune.

12. Government of India, 1965. Report of the Advisory Committee on the revision of list of SC and STs, Department of Social Security, New Delhi. Retrieved from https://tribal.nic.in/writereaddata/AnnualReport/LokurCommitteeReport. $p d f$

13. Government of India, 1991. National Sample Survey Organisation (NSSO) Report, Delhi.

14. Government of India, 2011. National Sample Survey Organisation (NSSO) Report, Delhi.

15. Government of India, 2012. Statistics on School Education 2010-11, MHRD, Bureau of Planning, Monitoring and Statistics, New Delhi. Retrieved from https://mhrd.gov.in/sites/upload_files/mhrd/files/statistics-new/SESSchool_2010-11.pdf

16. Government of India, 2013. Statistical Profiles of Scheduled Tribes in India 2013, Ministry of Tribal Affairs. Retrieved from http://www.tribal.nic.in/ST/StatisticalProfileof STs2013.pdf.

17. Government of India, 2018. Handbook on Social Welfare Statistics, Ministry of Social Justice and Empowerment, New Delhi.

18. Government of India \& U.P. 2011. District Census Handbook Ballia (PCA) Uttar Pradesh series 10 Part XII B, p 68-73.

19. Government of U.P. 2017. Statistical Diary Uttar Pradesh, Economics and Statistics Division, State Planning Institute Planning Department, Uttar Pradesh, Lucknow

20. https://shodhganga.inflibnet.ac.in/bitstream/10603/152197/13/12_chapter\%207.pdf

21. Hasnain, 2004. Indian Society and Culture: Continuity and Change, Jawahar Publishers \& Distributors, New Delhi.

22. Human Rights Watch, 2014. 'They Say We're Dirty': Denying an Education to India's Marginalized, April 2014, p.3.

23. India Human Development Survey (IHDS) Survey (2004-05). National Council of Applied Research, Delhi.

24. Jaiswal, A. 2017. Kharwar: A Dynamics of Change, Alfa Publications, New Delhi.

25. Jayaswal, M., Sinha, S.K., Kumari, A.K. and Arora, A. 2003. Parental support and academic achievement in tribal school students of Jharkhanda, Journal of All India Association for Educational Research, 15(3): 9-16.

26. Jha, J. and Jhingan, D. 2002. Elementary Education for the Poorest and Other Deprived Groups: The Real Challenge of Universalization, Centre for Policy Research, New Delhi.

27. NIRD Report (2011-12). Rural Development Statistics, National Institute of Rural Development retrieved from http://www.nird.org.in/Rural\%20Development\%20Statistics\%202011-12/data/sec-10.pdf

28. Pradhan, S.K. 2011. 'Problems of Tribal Education in India' Kurukshetra, 59(7): 26-31.

29. Rai, R.K. 2017. Socio-political aspects of tribal community: A case study of five districts (Ballia, Mau, Gorakhpur, Deoria, Maharajganj) of Purvanchal in Uttar Pradesh, International Journal of Current Research, 9(1): 45182-45188.

30. Rai, R.K. 2018. Tribes of Uttar Pradesh: Brief Introduction, IJAR, 4(1): 1143-1149.

31. Registrar General of India, 2001. Uttar Pradesh Data Highlights: The Scheduled Tribes, Census of India, 2001, Lucknow.

32. Singh, A.K. 1997. Endangered Tribals in India: Booby trap of development, Social Change, 27. 
